\title{
Research Progress of Phase-change Cooling in High-power LED Microchannel
}

\author{
Guanglin Liu", a, Wei Zhang ${ }^{1, b}$, Yuanzhi Sun ${ }^{1, c}$ \\ ${ }^{1}$ The Beijing Key Laboratory of Multiphase Flow and Heat Transfer for Low Grade Energy Utilization, \\ North China Electric Power University, Beijing, 102206, China \\ aliu0513@126.com, bw.zhang@ncepu.edu.cn, , c419157895@qq.com
}

\begin{abstract}
Keywords: phase-change cooling; microchannel; heat transfer enhancement
Abstract. In view of high-power LED microchannel phase-change cooling system, scientific problems, such as boiling incipience, flow pattern, heat transfer character, pressure drop and heat transfer instability are summarized. Proposing simultaneous measurement and analysis of phase-change cooling flow pattern, temperature response and synchronous pressure to reveal the mechanism of heat transfer instability, so as to development new method to inhibit heat transfer instability. Thermal siphon principle used for phase-change condensation for microchannel heat transfer is directed against the scientific problems of large consumption of pump power. And establish the optimized matching principle by optimization of property of system working fluid, nano particles, height difference between evaporation entrance and condensation outlet for phase-change condensation heat transfer of high-power LED microchannel.
\end{abstract}

\section{Introduction}

LED (Light Emitting Diode) lighting has been widely used, it has the advantages of energy saving, high efficiency, small size, long service life, quick response, low driving voltage, shock resistance and so on. The energy consumption is only $10-20 \%$ of the incandescent lamps under the same conditions of light effect. LED will be applied to lighting in large scale with the lower prices and improved luminous efficiency at advent of new processing technologies. It has become a hot topic in the field of high heat flow electronic chip cooling in recent years. But the typical area package is $1 \mathrm{~mm}^{2}$ and thermal power is $1-5 \mathrm{w}$ for high power LED chip, the heat flux generated in such a small area up to $100-500 \mathrm{w} / \mathrm{cm}^{2}$. The PN junction temperature of the LED will jump if the high heat flux does not release timely, and resulting in light emitting efficiency cut down, changing color temperature and color coefficient of LED chip and decreasing stability and life etc. Therefore, LED cooling has become one of the key issues of high power LED technology and industrial development.

Phase-change heat transfer in the microchannel studying is mainly for boiling incipience, flow pattern, heat transfer character, pressure dropping, instability and critical flux density, and it cannot master the mechanism of phase-change heat transfer in microchannel because of independence of the research. Since the typical size of the microchannel is at 10-1000 microns, the pump will consume relatively large pump power when the fluid flows through such a small channel. This paper is mainly summarized from the above aspects, summarizes the latest research, existing problems and corresponding research programs, to provide ideas for the design and operation of the microchannel phase-change heat for high-power LED cooling.

\section{Boiling Incipience}

Boiling temperature directly determines the start-up over temperature of boiling system, and the studies have shown that the boiling temperature can even reach 0.9Tc (Tc is the limit of superheat temperature) in the microchannel made by MEMS technique, the roughness of microchannel surface can reach $6.5 \mathrm{~nm}$, which is closed to critical nucleation radius [1], so the high start-up over temperature makes the LED chip burned down before the phase-change heat transfer microchannel cooler system operation. Channel section shape, geometric scale, quality flow and entrance 
undercooling temperature of cooling medium and surface wettability factors have an important effect on boiling incipience [2], so the initial boiling temperature prediction model should be fully considered above factors.

The control of boiling temperature in microchannel mainly includes active control and passive control technical methods. An active method was propose, namely seed bubble generator integrated in the microchannel, which using external pulse circuit to control the nucleation of periodic bubble generator, and this method could achieve the controlling of boiling temperature in the microchannel [3]. Passive methods include preparation of artificial nucleation sites [4], changeing channel surface roughness [5] and change of channel surface wettability [6].

\section{Flow pattern}

The temporal and spatial distribution structure of gas-liquid two-phase flow in microchannel leads to different flow pattern with different heat transfer characteristics and instability characteristics. The flow pattern in microchannel is mainly acquired by combination of high-speed camera and microscope. Due to the internal surface tension of microchannel plays a dominant role, and the effect of gravity is relatively weak, so it is generally considered that there is no stratified flow in microchannel. It has been found that flow pattern in the microchanenl are bubble flow, slug flow , annular flow and elongated bubble flow [7].

Two-phase general flow patterns and criteria for flow regime transition in microchannel. Due to different heat transfer characteristics corresponding to different flow patterns, it is great scientific significance to predict flow patterns and flow regime transitions according to operation of microchannel phase change heat exchangers in the microchannel.

Although studies have attempted to establish a general microchannel heat transfer flow pattern and dimensionless criteria of flow pattern conversion [8], but due to the researchers use different test piece and the cooling medium, so the results are not comparable.

\section{Heat Transfer Characteristics}

Compared with the phase change heat transfer on macro scale and micro channel, it was also found there are forced convection boiling and nucleate boiling mode [9]. In addition, Thome propose evaporation heat transfer model of the film according to the particularity of phase-change heat transfer in the micro channel [10], the theory is once the bubble will soon grow up, then the wall surface of the channel formed by the spatial limit of long bubbles because the channel scale is small. And the heat transfer efficiency of thin film evaporation between the bubble and the wall is high, which is the main mechanism of heat transfer in the microchannel.

In fact, the three types of heat transfer modes (forced convection boiling, nucleate boiling and liquid film evaporation) depend on the experimental elements, the experimental working fluids and the operating parameters. The researcher have also conducted original research in the microchannel heat transfer area, this paper presents a new heat transfer model of seed bubble generator control, the method is changing the external pulse to control generation frequency of seed bubble in microchannel, and control of evaporation interface within the channel. The microchannel phase-change heat transfer has controllable boiling initial temperature, higher heat transfer coefficient and uniform temperature distribution [11].

The heat transfer characteristics have an important influence on the critical heat flux in microchannel. The research of the critical heat flux in microchannel is mainly focused on the mechanism of heat transfer deterioration and the prediction model [12,13]. Studies have shown that in the micro channel heat transfer deterioration can be attributed to the phase-change heat transfer instability deterioration (dynamic heat transfer deterioration) and critical heat flux cause higher degree of steam which leads to heat transfer deterioration (static heat transfer deterioration). For the heat transfer deterioration caused by heat transfer instability, and the critical heat flux can be improved by suppressing the instability. 


\section{Heat transfer instability}

Instability is one of the key technical bottlenecks of phase-change heat exchangers for microchannel. The microchannel heat transfer instability is in the form of conversion of pulsation, the wall temperature changing of flow pattern and the channel inlet and outlet pressure vibration (or pressure drop pulsation on both sides of the channel). It has been found microchannel instability is characterized by long cycle/large amplitude ripple or short cycle/small amplitude ripple and irregular pulse [14].

Ledinegg instability [15], i.e. static instability, it occurs mainly in the negative slope region of the flow and pressure drop curves. For the heat input constant of microchannel, when the entrance flow rate decreases, the resistance of the liquid phase decreases, and when the flow is reduced to a certain extent, boiling in the microchannel occurs, result in the increase of two-phase frictional pressure drop. As a result, the pressure of the whole channel increases with the decrease of the flow rate.

As the flow rate continues to decrease, the increase of two-phase frictional resistance is smaller than the decrease of pressure drop caused by the decrease of flow rate, and the total pressure drop will decrease. Considering the pump drive characteristics of mircochannel boiling heat transfer, in the negative slope area of the flow rate and pressure drop, reducing the flow rate will cause the increase of pressure drop, so as to further reduce the flow, until the system runs in high dry zone, and then the system may be breakdown. The presence of throttling components at the channel entrance can significantly inhibit the occurrence of Ledinegg instability.

Pressure drop pulsation [16], generally speaking when the channel diameter ratio length is more than 150 , the channel is easy to produce compressed volume, and shape pressure drop type oscillation. Compared with macro scale, microchannel has relatively large diameter to length ratio, and more prone to shape pressure drop pulsation. In addition, the microchannel cross-sectional area is smaller, and there is a slight compressible space of $1 \mathrm{~mm} 3$ magnitude at the upstream pipe joint, which results in pressure drop pulsation in the channel. The pressure drop pulsation has the characteristics of long period and large amplitude.

Pulsation caused by nucleation and growth of bubbles [16]. At the macroscopic scale, the size of a single bubble is generally smaller than the boiling space scale, and the fluctuation caused by the nucleation and growth of bubbles is not significant. At the micro scale, the bubbles grow rapidly to equivalent scale, and even block the cross section of the channel. The pulsation produced by the bubble growth can not be neglected in the microchannel. Studies have shown that the pressure pulse produced by the nucleation of bubbles in the microchannel can even cause reflux in the channel. The magnitude of the pressure pulse generated by the nucleation is closely related to the superheat of the liquid in the channel prior to the nucleation of the bubble. The more degree of superheat, the more instability caused by the nucleation of bubbles.

Unstable in parallel channel [17].In parallel microchannels, the pressure of the inlet and outlet can be stable, but there are differences in the nucleation sites (such as the number of nucleation holes and nucleation sites). Therefore, the bubble nucleation and growth characteristics also showed differences, the resistance of different channels are different, resulting in uneven distribution of different fluid channels, the heat transfer coefficient and wall temperature characteristics will vary in different channels. The instability of parallel channel is dominated by the density fluctuation in the channel and the feedback action between different channels. It usually occurs in the condition of higher gas voids at the outlet, and has the characteristics of short cycle and small amplitude.

Suppression method of microchannel heat transfer instability. The main factors influencing the phase-change heat transfer microchannel instability including: entrance channel undercooling, upstream compressible volume in the channel, boiling starting temperature, channel geometry structure and system operation pressure and so on. Previous research shows that improving the system operating pressure [18], installation entrance throttling device [19], reducing the liquid subcooling and preparation of artificial nucleation point in the channel [20] and other measures could eliminate or significantly inhibit the phase-change heat transfer instability in microchannel. 


\section{Conclusions}

Microchannel heat exchanger not only has the advantages of compact structure, large specific surface area, high heat transfer efficiency, but also easy to integrate with LED chips. This paper summary the characteristics of start-up temperature and instability for heat transfer microchannel, which studied by adding nano fluid and operating parameters, and propose combination active artificial seed bubble and passive artificial nucleation ideas for reduced start-up temperature and reduce instability. Propose research area, such as thermal siphon principle used for phase change condensation for microchannel heat transfer is directed against the scientific problems of large consumption of pump power, and analysis and optimization of property of system working fluid, nano particles, height difference between evaporation entrance and condensation outlet. And establish the optimized matching principle for phase condensation heat transfer of high-power LED. At the same time, simultaneous measurement and analysis of the phase-change cooling, temperature response and synchronous pressure in the microchannel to reveal the mechanism of heat stability, so as to development a new method of inhibit heat transfer instability.

\section{Acknowledgements}

This work was financially supported by the National Natural Science Foundation of China ( 51476057), Fundamental Research Funds for the Central Universities (No.2015MS40, 2015MS47)

\section{References}

[1] L.W. Lin: Microscale Thermophys. Eng. Vol. 2 (1998), P. 71-85

[2] J. Li, P. Cheng: Int. J. Heat Mass Transfer Vol. 47 (2004), P. 2689-2698

[3] G.H. Liu, J.L. Xu and Y.P. Yang: Microfluid Nanofluidics Vol. 8 (2009), P. 341-359

[4] Y. Sun, L. Zhang and H. Xu: Int. J. Therm Sci Vol. 50 (2011), P. 881-889

[5] S.G. Kandlikar: Exp. Therm Fluid Sci Vol. 30 (2006), P. 441-447

[6] C. Choi, J.S. Shin, D. Kim: Exp. Therm Fluid Sci Vol. 35 (2011), P. 816-824

[7] R.Charnay, J. Bonjour, R. Revellin: Int. J. Heat Mass Transfer Vol.46 (2014), P. 1-16

[8] S. Haase: Int. J. Multiphase Flow Vol. 88 (2017), P. 251-269

[9] J.L. Xu, S. Shen, Y.H. Gan: J Micromech Microeng Vol. 15 (2005), P. 1344-1361

[10] R. Thome, V. Dupont, A. M. Jacobi: Int. J. Heat Mass Transfer Vol. 47 (2004), P. 3375-3385

[11] G.H. Liu, J.L. Xu, W. Zhang: Int. J. Multiphase Flow Vol. 35 (2009), P. 773-790

[12] A. Kosar: Int. J. Therm SCI Vol. 48 (2009), P. 261-270

[13] C.R. Khara, I. Mudawar, M.M. Hasan: Int. J. Heat Mass Transfer Vol. 55 (2012), P. 4154-4168

[14] S.K. Saha, G.P. Celata: Instability in flow boiling in microchannels (Springer, Germany 2016).

[15] T.J. Zhang, T. Tong, J.Y. Chang: Int. J. Heat Mass Transfer Vol. 52 (2009), P. 5661-5674

[16] M. Asadi, G. Xie B. Sunden: Int. J. Heat Mass Transfer Vol. 79 (2014), P. 34-53

[17] Y.Y. Jiang, H. Osada, M. Inagaki: Int. J. Heat Mass Transfer Vol.56 (2013), P. 640-652

[18] C.Y. Kuo, Y. Pele: Int. J. Heat Mass Transfer Vol. 52 (2009), P. 271-280

[19] G.D. Wang, P. Cheng, A.E. Bergles: Int. J. Heat Mass Transfer Vol. 51 (2008), P. 2267-2281

[20] C.J. Kuo, Y. Peles: J. Heat Transfer Vol. 130 (2008), P. 351-358 